\title{
Complete Genome Sequence of Enterobacter roggenkampii Strain KQ-01, Isolated from Bacterial Wilt-Resistant Mulberry Cultivar YS283
}

\author{
Yinan Zhou, Hongyu Yang, and Jiping Liu ${ }^{\dagger}$ \\ College of Animal Science, South China Agriculture University, Wushan Road, Guangzhou, Guangdong, \\ 510642, China
}

\begin{abstract}
An Enterobacter roggenkampii strain, named KQ-01, was isolated for the first time from the diseased roots of the bacterial wilt-resistant mulberry (Morus atropurpurea) cultivar YS283 in the western part of Guangxi Zhuang Autonomous Region, China. E. roggenkampiiKQ-01 was characterized by a single, circular chromosome of 4,667,006 bp in size, with a $56.16 \%$ GC content. In total, 4,396 genes were annotated, of which 4,281 were assigned as proteincoding genes. In addition, 25 ribosomal RNA, 84 transfer RNA, and 6 noncoding RNA genes as well as 34 pseudogenes were predicted. The resource herein presented will help to explore the pathogenetic mechanisms of mulberry wilt disease caused by genus Enterobacter.
\end{abstract}

Mulberry is a perennial woody plant widespread in Asia, Africa, and Europe, of high economic value (Dhanyalakshmi and Nataraja 2018; Rodrigues et al. 2019). In 2008, bacterial wilt of mulberry caused by the Enterobacter cloacae complex (ECC) was first reported in China (Wang et al. 2008). Later, the genome sequence of E. mori LMG 25706 was published (Zhu et al. 2011). Although ECC represents a serious threat for most mulberry varieties, the bacterial wilt-resistant $M$. atropurpurea ' $Y S 283$ ' is believed to be resistant to bacterial wilt disease. Since 1993, M. atropurpurea 'YS283' has been selected and bred in Guangdong, China (Huang 1993), and successfully commercialized in the country due to its excellent resistance to many pathogenic bacteria such as Ralstonia solanacearum and related species (Dai et al. 2016). However, wilting symptoms in M. atropurpurea 'YS283' have been increasingly observed in Guangxi, South China, which represents the largest sericulture production site in China. The wilting symptoms of the mulberry, which appeared to be caused by a bacterial infection, were confirmed in our laboratory. To boost research on etiology and pathogenesis of such bacterial infection, we isolated one of the pathogenic bacteria from the infected mulberry (namely, E. roggenkampii KQ-01) and sequenced and assembled its genome. E. roggenkampii KQ-01 culture has been deposited in the International Depositary Authority (Guangdong Microbial Culture Collection Center, Guangzhou, China) under accession number GDMCC 1.6104.

During summer 2017, E. roggenkampii strain KQ-01 was isolated from the infected roots of bacterial wilt-resistant mulberry ( $M$. atropurpurea) cultivar YS283, found in Guangxi Zhuang Autonomous Region, China $\left(109^{\circ} 26^{\prime} 13^{\prime \prime} \mathrm{E}, 24^{\circ} 52^{\prime} 85^{\prime \prime} \mathrm{N}\right)$. To isolate the pathogenic bacteria, the infected roots were washed with sterilized water prior to cutting them into $50-\mathrm{mm}$ long sections. Sections were soaked in $75 \%$ ethyl alcohol for $15 \mathrm{~s}$ and incubated with sterilized water in a rotary shaker at $130 \mathrm{rpm}$ for $30 \mathrm{~min}$ at $25^{\circ} \mathrm{C}$. After a gradient dilution, the supernatant was placed on a cultural plate with triphenyl tetrazolium chloride (TTC) medium and incubated at $30^{\circ} \mathrm{C}$ and $75 \%$ humidity for $72 \mathrm{~h}$. Single colonies were picked up and

\footnotetext{
${ }^{\dagger}$ Corresponding author: J. Liu; liujiping@scau.edu.cn
}

The author(s) declare no conflict of interest.

Accepted for publication 21 August 2020
Funding

This work was financially supported by Agriculture Research System of China (CARS-18- ZJ0304).

\section{Keywords}

bacterial wilt, complete genome, Enterobacter roggenkampii KQ-01, mulberry cultivar YS283 
Table 1. Genome of Enterobacter roggenkampii strain KQ-01

$\begin{array}{lr}\text { Features }^{\text {a }} & \text { Numbers } \\ \text { Genes } & 4,396 \\ \text { Protein-coding genes } & 4,281 \\ \text { Transfer RNAs } & 84 \\ \text { Ribosomal RNAs } & 25 \\ \text { Noncoding RNAs } & 6 \\ \text { Pseudogenes } & 34 \\ \text { Hypothetical proteins } & 429 \\ \text { Proteins with COG assignments } & 3,903 \\ \text { Proteins with GO assignments } & 1,018 \\ \text { Proteins with KEGG assignments } & 885 \\ \text { Predicted Gls } & 36\end{array}$

a $\mathrm{COG}=$ cluster of orthologous groups, $\mathrm{GO}=$ Gene Ontology, KEGG = Kyoto Encyclopedia of Genes and Genomes, and Gls = genomic islands.

streaked in TTC medium. Monoclonal colonies were selected and transferred onto a new plate with TTC medium for pure culture.

For quality assurance, genomic DNA was extracted and purified from a pure culture of a single bacterial isolate of $E$. roggenkampii KQ-01, using the Bacteria Genomic DNA Kit (CoWin Biosciences, Beijing, China). The quantity and quality of the DNA were assessed by a Qubit 2.0 Fluorometer (Thermo Fisher Scientific, Waltham, MA, U.S.A.). Prior to DNA sequencing, the $16 \mathrm{~S}$ ribosomal DNA (rDNA) sequence obtained was compared with those deposited in NCBI GenBank using the NCBI BLAST algorithm. For further study of the genetic background, the complete genome sequence was obtained and analyzed. For paired-end sequencing, whole-genome DNA was used to prepare a DNA library with a 350-bp insert. Deep sequencing (depth $=440 \times$ ) was conducted with manufactured $2 \times 150$-bp alignment reads on Illumina Hiseq4000 System by Science Corporation of Gene Technology Co., Ltd. (Guangzhou, China) and, in total, 6,846,906 paired-end reads were obtained. Genome sequences were assembled using SPAdes, version 3.5.0 (Bankevich et al. 2012). The genome sequence data were uploaded to Similar Genome Finder Server (Davis et al. 2020) to determine the accurate and reliable taxonomic position. Phylogenomic analysis was performed by average nucleotide identity based on BLAST+ (ANIb) (Richter et al. 2016). The genome was automatically annotated using the NCBI Prokaryotic Genome Annotation Pipeline (Haft et al. 2018) and the RAST tool kit (Overbeek et al. 2014). Functional analysis was done by using clusters of orthologous groups (COGs) of proteins (Galperin et al. 2015), Gene Ontology (GO) (Ashburner et al. 2000), and Kyoto Encyclopedia of Genes and Genomes (KEGG) pathways (Kanehisa et al. 2016) annotation. Identification of genomic islands (GIs) was performed using the IslandViewer 4 platform and the SIGI-HMM, IslandPick, IslandPath-DIMOB, and Islander prediction methods (Bertelli et al. 2017).

E. roggenkampii KQ-01 was first identified as ECC based on the 16S rDNA sequence. In addition, the genome sequence was highly identical $(99.10 \%)$ to E. roggenkampii SMART_ 269.v1 (NCBI accession number GCA_001471995) isolated from Homo sapiens based on ANIb analysis. The complete genome of E. roggenkampi KQ-01 was composed of a single, continuous, circular chromosome of 4,667,006 bp in size, with a $56.16 \%$ GC content and no plasmids. Moreover, in total, 4,396 genes were annotated in the E. roggenkampii KQ-01 strain genome, of which 4,281 genes were assigned as putative protein-coding genes. In addition, 115 RNA-encoding genes, including 25 ribosomal RNA, 84 transfer RNA, and 6 noncoding RNA genes as well as 34 pseudogenes were predicted. By using functional protein alignments, 3,903 proteins were assigned to the COG database, 1,018 proteins were assigned to GO terms, and 885 proteins were mapped to KEGG pathways. By using the IslandViewer 4 platform, 36 Gls were identified. The annotated features are summarized in Table 1.

To the best of our knowledge, this is the first reported genome of E. roggenkampii KQ-01 isolated from mulberry. Our data provide a starting platform for comparative genomics of other Enterobacter spp., which are responsible for mulberry wilt disease in the main region of sericulture, and clarify the pathogenesis of E. roggenkampii KQ-01 in mulberry. 
Data availability. The genome sequence of E. roggenkampii strain KQ-01 has been deposited in NCBI GenBank in BioProject number PRJNA644454 under accession number CP058637. The original data have been deposited in the NCBI Sequence Read Archive under accession number SRX8696658.

\section{Literature Cited}

Ashburner, M., Ball, C. A., Blake, J. A., Botstein, D., Butler, H., Cherry, J. M., Davis, A. P., Dolinski, K., Dwight, S. S., Eppig, J. T., Harris, M. A., Hill, D. P., Issel-Tarver, L., Kasarskis, A., Lewis, S., Matese, J. C., Richardson, J. E., Ringwald, M., Rubin, G. M., and Sherlock, G. 2000. Gene Ontology: Tool for the unification of biology. Nat. Genet. 25:25-29.

Bankevich, A., Nurk, S., Antipov, D., Gurevich, A. A., Dvorkin, M., Kulikov, A. S., Lesin, V. M., Nikolenko, S. I., Pham, S., Prjibelski, A. D., Pyshkin, A. V., Sirotkin, A. V., Vyahhi, N., Tesler, G., Alekseyev, M. A., and Pevzner, P. A. 2012. SPAdes: A new genome assembly algorithm and its applications to single-cell sequencing. J. Comput. Biol. 19:455-477.

Bertelli, C., Laird, M. R., Williams, K. P., Lau, B. Y., Hoad, G., Winsor, G. L., and Brinkman, F. S. L. 2017. IslandViewer 4: Expanded prediction of genomic islands for larger-scale datasets. Nucleic Acids Res. 45:W30-W35.

Dai, F., Wang, Z., Luo, G., and Tang, C. 2016. Transcriptional analysis of different mulberry cultivars in response to Ralstonia solanacearum. Can. J. For. Res. 46:152-162.

Davis, J. J., Wattam, A. R., Aziz, R. K., Thomas, B., Ralph, B., Butler, R. M., Philippe, C., Conrad, N., Dickerman, A., Dietrich, E. M., Gabbard, J. L., Gerdes, S., Guard, A., Kenyon, R. W., Machi, D., Mao, C., Murphy-Olson, D., Nguyen, M., Nordberg, E. K., Olsen, G. J., Olson, R. D., Overbeek, J. C., Overbeek, R., Parrello, B., Pusch, G. D., Shukla, M., Thomas, C., VanOeffelen, M., Vonstein, V., Warren, A. S., Xia, F., Xie, D., Yoo, H., and Stevens, R. 2020. The PATRIC bioinformatics resource center: Expanding data and analysis capabilities. Nucleic Acids Res. 48:D606-D612.

Dhanyalakshmi, K. H., and Nataraja, K. N. 2018. Mulberry (Morus spp.) has the features to treat as a potential perennial model system. Plant Signal. Behav. 13: e1491267.

Galperin, M. Y., Makarova, K. S., Wolf, Y. I., and Koonin, E. V. 2015. Expanded microbial genome coverage and improved protein family annotation in the COG database. Nucleic Acids Res. 43:D261-D269.
Haft, D. H., DiCuccio, M., Badretdin, A., Brover, V., Chetvernin, V., O'Neill, K., Li, W., Chitsaz, F., Derbyshire, M. K., Gonzales, N. R., Gwadz, M., Lu, F., Marchler, G. H., Song, J. S., Thanki, N., Yamashita, R. A., Zheng, C., Thibaud-Nissen, F., Geer, L. Y., Marchler-Bauer, A., and Pruitt, K. D. 2018. RefSeq: An update on prokaryotic genome annotation and curation. Nucleic Acids Res. 46: D851-D860.

Huang, F. 1993. Breeding of Morus atropurpurea cultivar 'YS283' for bacterial wiltresistant, high-yielding and high-quality. Guangdong Sericulture 04:32-36+20 (In Chinese).

Kanehisa, M., Sato, Y., Kawashima, M., Furumichi, M., and Tanabe, M. 2016. KEGG as a reference resource for gene and protein annotation. Nucleic Acids Res. 44: D457-D462.

Overbeek, R., Olson, R., Pusch, G. D., Olsen, G. J., Davis, J. J., Disz, T., Edwards, R. A., Gerdes, S., Parrello, B., Shukla, M., Vonstein, V., Wattam, A. R., Xia, F., and Stevens, R. 2014. The SEED and the rapid annotation of microbial genomes using subsystems technology (RAST). Nucleic Acids Res. 42:D206-D214.

Richter, M., Rosselló-Móra, R., Glöckner, F. O., and Peplies, J. 2016. JSpeciesWS: A web server for prokaryotic species circumscription based on pairwise genome comparison. Bioinformatics 32:929-931.

Rodrigues, E. L., Marcelino, G., Silva, G. T., Figueiredo, P. S., Garcez, W. S., Corsino, J., Rita de Cássia, A. G., and Karine de Cássia, F. 2019. Nutraceutical and medicinal potential of the Morus species in metabolic dysfunctions. Int. J. Mol. Sci. 20:301.

Wang, G. F., Praphat, K., Xie, G. L., Zhu, B., Li, B., Liu, B., and Zhou, Q. 2008. Bacterial wilt of mulberry (Morus alba) caused by Enterobacter cloacae in China. Plant Dis. 92:483.

Zhu, B., Zhang, G.-Q., Lou, M.-M., Tian, W.-X., Li, B., Zhou, X.-P., Wang, G.-F., Liu, H., Xie, G.-L., and Jin, G.-L. 2011. Genome sequence of the Enterobacter mori type strain, LMG 25706, a pathogenic bacterium of Morus alba L. J. Bacteriol. 193:3670-3671. 